\title{
Iron-deficiency Anemia in Egyptian Type 1 Diabetic Children at Suez Canal University Hospital
}

\section{Enas F Elngar ${ }^{1}$, Hassan A Shora ${ }^{2 *}$, Noran Bayoumi ${ }^{1}$, Sylvias W Boulos ${ }^{1}$ and Enas S Abbas ${ }^{3}$}

${ }^{1}$ Faculty of Medicine, Suez Canal University, Ismailia, Egypt

${ }^{2}$ Port-Said University and National Nutrition Institute, Cairo, Egypt

${ }^{3}$ National Nutrition Institute, Cairo, Egypt

*Corresponding Author: Hassan A Shora, Senior Research Scientist Head of

Medicine and Diabetes Center Port-Said University and Ismailia General Hospital,

Egypt.
Received: February 26, 2021

Published: March 11, 2021

(C) All rights are reserved by Hassan A Shora., et al.

\section{Abstract}

Aim: The aim of this study is to determine how common iron deficiency anemia among children with type 1 diabetes for early adequate treatment aiming for improving their quality of life.

Objectives: To find out a relationship between hemoglobin concentration and HbA1c and any correlation between HbA1c, serum ferritin and serum iron level. To detect a correlation between serum ferritin, serum iron and duration of diabetes.

Methods: This study is an observational cross sectional study, carried out on 90 children aged from 3 to 17 years old, already diagnosed by type 1 diabetes, according to diagnostic criteria of American Diabetes Association. Hb A1c $\geq 6.5 \%$ Detailed medical history and full clinical examination were done, Investigations included complete blood picture, Hba1c, serum ferritin, serum iron.

Results: Prevalence of iron deficiency anemia among type 1 diabetic children was $56.7 \%$. The mean of HbA1c is $7.04 \%$ in anemic diabetic patients while the mean is $7.81 \%$ in asymptomatic diabetic patients and there is a significant difference between them. There is a statistically significant correlation between $\mathrm{HbA1c}$ and $\mathrm{Hb}$, serum iron and serum ferritin levels in type 1 diabetic patients There was a weak positive correlation between duration of diabetes and mean Hb levels, serum iron levels, and serum ferritin levels. Conclusion: The prevalence of iron deficiency anemia was high among Egyptian type 1 diabetic children, and value of HbA1c was higher among severe iron deficiency anemic patients than moderately iron deficiency anemic patients.

Keywords: Anemia; Iron; Type1 Diabetes

\section{Introduction}

The term diabetes mellitus DM is a complex metabolic disorder characterized by persistent hyperglycemia that occurs because of deficiency in insulin secretion, insulin activity, or both. That prompts variations from the normal blood glucose with profound impacts on carbohydrate metabolism It is a rapidly developing non-communicable disease all over the world and is one of the most well-known endocrine disease in children around the world [1].

Long-term diabetic complications are classified into microvascular (microangiopathy of micro vessels) and macrovascular (macroangiopathy of macro vessels). Microvascular complications include the eyes (retinopathy) resulting in visual impairment or even loss, the kidneys leading to Diabetic Kidney Disease and nerves 
(neuropathy) leading to diabetic foot a consequent of peripheral arterial disease with extreme ischemia with potential amputation. Macrovascular complications include diabetic cardiomyopathy in addition to increased risk of infections that may be complicated by , for example, respiratory failure, strok and periphreral arterial disease.

Diabetes does not directly cause anemia, but certain complications and conditions related to diabetes can contribute to it as nephropathy and neuropathy [3]. Diabetes frequently leads to nephropathy and this will cause anemia as the kidneys secret a hormone called erythropoietin (EPO), which stimulates the bone marrow to generate red blood cells. Diabetic kidneys don't secret sufficient EPO to keep up with body needs [4]. Autonomic neuropathy in diabetic patients hinders the body's capacity to legitimately stimulate the kidneys to create more erythropoietin in reaction to anemia [5].

Iron deficiency is characterized by decreased red blood cell (RBC) mass transporting oxygen and carbon dioxide which disables the body's capacity for gas exchange [6]. Among the conceivable components that decline kidney function, is renal ischemia caused by a decreased oxygen supply due to decreased levels of $\mathrm{Hb}$ and diabetic cardiomyopathy [5]. Diabetic nephropathy is rare among young type 1 diabetic children.

Iron deficiency anemia is the foremost common type and is effectively treatable by dietary changes and oral or parentral iron and other supplements. It is an important public health problem in the Eastern Mediterranean countries. It is estimated that more than one third of the population in Middle East have iron-deficiency anemia. Pregnant ladies and young children are at utmost risk; approximately $50 \%$ of pregnant ladies and $67.4 \%$ of children under- 5 and $15.5 \%$ school adolescents have iron deficiency anemia. WHO classified Egypt as direct risk of iron deficiency anemia among preschool children as its predominance was $29.9 \%$ [7]. Iron plays an essential role in hemoglobin $(\mathrm{Hb})$ synthesis, electron transport for cellular respiration, DNA synthesis, and other vital enzymatic reactions [8].

As iron deficiency anemia in patients with type 1 diabetes leads to fatigue and impairs cognitive function as well as the ability to work, thus influencing the psychosocial development of children with type 1 diabetes. So early detection and treatment of it improves quality of life among children [9]. The use of HbA1c for diagnosing and controlling glycemia in type 1 diabetic children is controversial and has pitfalls spectacularly in low-diagnostic threshold. The relationship between anemia and HbA1c is complicated and further studies are needed to resolve that issue. Most available studies focused on type 2 adult diabetics while few studies were conducted in Egypt in children with type 1 diabetes mellitus.

\section{Subjects and Methods}

This study was an observational cross-sectional study. The study was carried out in Pediatric Diabetic and Endocrine Metabolic outpatient clinic in Suez Canal University hospital. A random sample of children who were already diagnosed as type 1 diabetics in the Suez Canal university hospital were included in the study. Written informed consents were obtained from parents or caregivers of children.

\section{Inclusion criteria}

Male and female children of age range: 3 years old to 17 years old already diagnosed as type 1 diabetics. (according to diagnostic criteria of American diabetic association (ADA)2020, that include the following: (FPG) level $\geq 126 \mathrm{mg} / \mathrm{dL}$ or A 2-hour plasma glucose level $\geq 200 \mathrm{mg} / \mathrm{dL}$ or A random plasma glucose $\geq 200 \mathrm{mg} / \mathrm{dL}$ in a patient with classic symptoms of hyperglycemia or hyperglycemic crisis) (guideline 2020).

\section{Exclusion criteria}

Children on iron treatment. children with congenital or hemolytic anemia (as thalassemia,........ect). Children with co-morbid diseases such as cardiac diseases, renal failure and chronic hepatitis. Diseases that can cause elevation of serum ferritin such as any inflammation, recent infection, malignancy and autoimmune diseases and obese children were excluded from the study.

Investigations include the following:

- Complete blood picture: Hemoglobin levels $(\mathrm{g} / \mathrm{dL})$ to diagnose anemia at sea level. Children were stratified by age: 5 -11 years $\geq 11.5$. Children, $12-14$ years $\geq 12$. 15 years and above $\geq 12$. Men, 15 years and above $\geq 13$.

- Hba1c: Was done by immunosorbent assays (ELISA). Collected plasma using EDTA as an anticoagulant. Centrifuge samples for 15 minutes at $1000 \times \mathrm{g}$ within 30 minutes of collection. Normal HbA1c level $<6.5 \%$.

Citation: Enas F Elngar, Hassan A Shora, Noran Bayoumi, Sylvias W Boulos and Enas S Abbas . "Iron-deficiency Anemia in Egyptian Type 1 Diabetic Children at Suez Canal University Hospital". Acta Scientific Medical Sciences 5.4 (2021): 67-73. 
- Serum ferritin: Was done by immunosorbent assays (ELISA). Serum should be prepared from a whole blood specimen obtained by acceptable medical techniques without additives. Serum ferritin concentrations normal range is 15300 go/l. Iron deficiency is considered when concentration of serum ferritin is $<13$ go / dl for children below 5 years old and $<15$ go /dl for children above 5 years old.

- Serum iron: Was measured by a modification of the automated AAII-25 colorimetric method, which was based on the procedures of Giovanelli., et al. and of Ramsey the method has been modified further to be performed on an Alkema Flow Solutions IV (rapid-flow analyzer) system. Iron was quantitated by measuring the intensity of the violet complex that was formed in the reaction between ferrozine and $\mathrm{Fe}++$ in acetate buffer at $562 \mathrm{~nm}$. Thiourea was added to complex $\mathrm{Cu}++$, which can also bind with ferrozine and yield falsely elevated iron. normal value 60 to $170 \mathrm{mcg} / \mathrm{dL}$.

Data was collected in Excel data sheet. Data was analyzed using Statistical Package for The Social Sciences (SPSS 21). Continuous variables were presented as mean \pm standard deviation (SD), and were compared using student's T test. Categorical variables were presented as frequencies and percentages and were compared using person's chi-square test. The results were presented in the appropriate forms of tables and graphs using Microsoft Excel. P value less than 0.05 was considered significant.

\section{Results}

Demographic data

Table 1 comparison between anemic and non-anemic diabetic children according to gender, residency and age.it showed that there were $30.8 \%$ males who were non-anemic compared to $45 \%$ males who were anemic while there were $69.2 \%$ females non anemic and $54.9 \%$ of females were anemic with no significant difference between them.

It shows that there were $38.5 \%$ urban diabetic children were non-anemic and 51\% were anemic while there were $61.5 \%$ rural diabetic children were found non-anemic and $49 \%$ were anemic with no significant deference between them. This subclassification was made due to differences in sociodemographic conditions between urban and rural children.
It was found that the mean age in non-anemic patents was 10.05 while mean age in anemic patients was 10.22 , with no significant deference between them.

Iron deficiency anemia was more prevalent among age 7- 11 years old diabetic children as it represents $41.2 \%$.

\begin{tabular}{|c|c|c|c|c|c|c|}
\hline \multirow{2}{*}{\multicolumn{2}{|c|}{ Title }} & \multicolumn{2}{|c|}{$\begin{array}{c}\text { Non- anemic } \\
\text { (39) }\end{array}$} & \multicolumn{2}{|c|}{ Anemic (51) } & \multirow[t]{2}{*}{ P-value } \\
\hline & & No. & $\%$ & No. & $\%$ & \\
\hline \multirow{2}{*}{ Gender } & Male & 12 & 30.80 & 23 & 45.10 & \multirow{2}{*}{$0.122 ¥$} \\
\hline & Female & 27 & 69.20 & 28 & 54.90 & \\
\hline \multirow{2}{*}{ Residence } & Urban & 15 & 38.50 & 26 & 51.00 & \multirow{2}{*}{$0.167 ¥$} \\
\hline & Rural & 24 & 61.50 & 25 & 49.00 & \\
\hline \multirow{6}{*}{$\begin{array}{l}\text { Age } \\
\text { (years) }\end{array}$} & $2-$ & 10 & 25.60 & 10 & 19.6 & \multirow{4}{*}{$0.175 ¥$} \\
\hline & 7- & 9 & 23.10 & 21 & 41.20 & \\
\hline & 11- & 14 & 35.90 & 10 & 19.60 & \\
\hline & $15-18$ & 6 & 15.40 & 10 & 19.6 & \\
\hline & & Mean & SD & Mean & SD & \multirow{2}{*}{$0.85 €$} \\
\hline & & 10.05 & 4.04 & 10.22 & 3.91 & \\
\hline
\end{tabular}

Table 1: Sociodemographic characters of the diabetics under the study.

$¥$ Fisher Exact test

\# Chi square test

$€$ student-t test.

Table 2 shows that the prevalence of iron deficiency anemia among type 1 diabetic children was $56.7 \%$.

\begin{tabular}{|c|c|c|c|c|}
\hline \multirow{2}{*}{ Title } & \multicolumn{2}{|c|}{ Non-anemic (39) } & \multicolumn{2}{c|}{ anemic (51) } \\
\cline { 2 - 5 } & No. & \% & No. & \% \\
\hline Total prevalence & 39 & 43.3 & 51 & 56.7 \\
\hline
\end{tabular}

Table 2: The prevalence of iron deficiency anemia.

Table 3 shows that the mean of HBA1c is $7.04 \%$ in non-anemic diabetic patients while the mean is $7.81 \%$ in anemic diabetic patients and there is a significant difference between them.

Table 4 shows that there is a moderate negative correlation between HBA1c and HB and serum iron while there is a weak negative correlation between HBA1c and serum ferritin. 


\begin{tabular}{|c|c|c|c|c|c|}
\hline \multirow{2}{*}{ Title } & \multicolumn{2}{|c|}{$\begin{array}{c}\text { Non-anemic } \\
\text { (39) }\end{array}$} & \multicolumn{2}{c|}{ Anemic (51) } & \multirow{2}{*}{$\begin{array}{c}\text { P-Value } \\
€\end{array}$} \\
\cline { 2 - 5 } & Mean & SD & Mean & SD & \\
\hline HBA1c \% & 7.04 & 1.50 & 7.81 & 1.93 & $0.04^{*}$ \\
\hline
\end{tabular}

Table 3: Difference of HBA1c regarding presence of anemia in the sample under the study.

$€$ Mann-Whitney test.

*Statistically significant at $95 \%$ level of confidence .

\begin{tabular}{|l|c|c|}
\hline \multirow{2}{*}{} & \multicolumn{2}{|c|}{ HBA1c \% } \\
\cline { 2 - 3 } & $\begin{array}{c}\text { Pearson correlation } \\
\text { coefficient (r) }\end{array}$ & P-value \\
\hline HB (g/dL) & -0.35 & $0.00^{*}$ \\
\hline Serum iron (go/l) & -0.38 & $0.00^{*}$ \\
\hline $\begin{array}{l}\text { Serum ferritin } \\
\text { (go/dl) }\end{array}$ & -0.22 & $0.04^{*}$ \\
\hline
\end{tabular}

Table 4: Correlation between $\mathrm{HbA1c}$ and $\mathrm{Hb}$, serum iron and serum ferritin levels in type 1 diabetic children

Pearson correlation.

*Statistically significant at $95 \%$ level of confidence.

There is a statistically significant correlation between HBA1c and $\mathrm{HB}$, serum iron and serum ferritin levels in type 1 diabetic patients.

Table 5 shows that there was a weak positive correlation between duration of diabetes and $\mathrm{Hb}$, serum iron levels, and serum ferritin levels $\mathrm{P} \leq .0 .04,0.68$ and 0.18 respectively.

\begin{tabular}{|l|c|c|}
\hline \multirow{2}{*}{} & \multicolumn{2}{|c|}{ Duration of diabetes } \\
\cline { 2 - 3 } & $\begin{array}{c}\text { Pearson correlation } \\
\text { coefficient (r) }\end{array}$ & P-value \\
\hline $\mathrm{Hb}(\mathrm{g} / \mathrm{dL})$ & 0.21 & $0.04^{*}$ \\
\hline Serum iron(go/l) & 0.04 & 0.68 \\
\hline Serum ferritin (go/dl) & 0.14 & 0.18 \\
\hline
\end{tabular}

Table 5: Correlation between duration of diabetes and serum iron, ferritin and $\mathrm{Hb}$ levels.

Pearson correlation.

*Statistically significant at $95 \%$ level of confidence.
There was a statistically significant correlation between duration of diabetes and $\mathrm{Hb}$ while there was no statistically significant correlation between duration of diabetes, serum iron and serum ferritin.

\section{Discussion}

This study was carried out to determine the prevalence of iron deficiency anemia in children with type 1 diabetes as it is one of the most common and preventable disease aiming to improve their quality of life.

As shown in table 1, iron deficiency anemia among type 1 diabetic children was more prominent among age group 7- 11 years $41.2 \%$, the mean of age in iron deficient diabetic patients was 10.22 years and that $54.9 \%$ of anemic patients were female while $45.1 \%$ were male this probably may be due to the menstruation in pubertal females. In our previous study we demonstrated that iron deficiency anemia was prevalent in $39.29 \%$ in children [10]. Furthermore, inadequate iron intake is the most common cause of IDA in Egyptian children especially in rural areas where poor socioeconomic conditions, parasitic infestations, ignorance and neglect of dietary requirements. The requirements are increased in this rapid growth in pubertal stage of development that are usually not met. Poor nutritional habits of dietary iron of animal sources was reported by a recent study among female Egyptian school children [11]. Type 1 diabetic children are amenable to recurrent episodes of diabetic ketoacidosis which leads to severe vomiting, anorexia, repeated hospitalization and infection in addition to extravascular hemolysis that leads to chronic blood loss. The diagnosis of iron deficiency anemia can be easily made by laboratory demonstration of iron deficient state or by evaluating body response to a therapeutic iron supplementation. Salah., et al. studied prevalence and type of anemia in young Egyptian patients with type 1 diabetes mellitus and found that more than two-thirds (70.7\%) of the anemic patients were post-pubertal, the mean age was 11.15 year and $50.7 \%$ of anemic patients were female while $49.3 \%$ were male, He referred the cause to the menstruation in pubertal females in addition to potential decreased iron intake that led to nutritional anemia [12]. The total prevalence of the iron deficiency anemia among type 1 diabetics was $56.7 \%$. this was consistent with that of Salah and his colleagues who found that the prevalence of iron deficiency anemia among pediatric type 1 diabetics was 54.7\% [13]. Akkermans and his colleges studied the iron status and its association 
with HbA1c levels in Dutch children with type 1diabetes mellitus and found that the prevalence of iron deficiency was $3.7 \%$ this is lower than in our study as it was observed on different population (Dutch children) with different socioeconomic and environmental conditions. Furthermore, they used different blood test (Zinc uroporphyrin) and used different working definition and classification of their patients into absolute, functional iron deficiency and iron deficiency anemia [14].

Tarim and his colleges studied the effects of iron deficiency anemia on hemoglobin A1c in type 1diabetic children in Uludağ University, Bursa, Turkey that study included 37 patients with type 1 DM, 11 non-diabetic subjects with iron deficiency anemia and 20 completely healthy subjects and found that the prevalence of iron deficiency among diabetics was $29.7 \%$ this prevalence was lower than our study as it was done in Turkey with different study population and use different classification to the subject included in the study [15]. Interpretation of increased HbA1c in this study was made in the context of decreased serum iron concentration which was markedly decreased in type 1 diabetic children.

Current study has shown that the mean of HbA1c in the anemic patients was $7.81 \%$ which was statistically significant compared to non-anemic patients which was $7.4 \%$ and that there was a negative correlation between hemoglobin, and both of serum iron and serum ferritin. However, serum ferritin may increase due to inflammatory diseases and other stimuli and its elevation doesn't exclude iron deficiency. So, it has limited diagnosing utility in this setting. It was recommended to test hs-CRP in inflammatory bowel disease to confirm the diagnosis of iron deficiency anemia. However, no consensus for adding hs-CRP in the diagnosis of IDA in the presence of increased serum ferritin. Moreover, hs-CRP is not included in all available guidelines to measure it in case of iron deficiency anemia with inflammatory diseases. That meant that the presence of iron deficiency anemia led to higher levels of HbA1c in diabetic children. Iron deficiency accelerates glycation of hemoglobin by changing the its molecular structure through increased peroxidation in diabetics. This may be a consequence of chronic low-grade inflammation in diabetics. Furthermore, it increases the life span of red blood cells. Patients with type 1 diabetes with IDA have a restricted availability of iron that is necessary for erythropoiesis due to increased hepcidin expression [16].
In a systematic review that included 47 students with iron deficiency anemia it was found that the mean $\mathrm{HbA1c}$ level decreased significantly from $6.15 \% \pm 0.62$ to $5.25 \% \pm 0.45$ after treatment with oral iron supplement for 20 weeks These results demonstrated that iron deficiency is associated with higher levels of HbA1C, which could cause problems in the interpretation and diagnosis of uncontrolled diabetes mellitus in iron deficient anemic patients [17].

Madhu S., et al. studied the effect of iron deficiency anemia and iron supplementation on $\mathrm{HbA} 1 \mathrm{c}$ levels for diagnosis of prediabetes and diabetes mellitus in Asian Indians, the subjects were clustered in 2 groups, iron deficient anemia (IDA) and healthy controls (HC) and found that the mean levels of RBC Indices and iron study parameters were significantly higher $(\mathrm{p}<0.001)$ in IDA group as compared to HC and the mean glycated hemoglobin \% levels in the pre-intervention arm in the IDA subjects were significantly higher by $0.66 \%(p<0.001)$ as compared to the healthy controls [18].

Intra., et al. studied the effects of iron deficiency anemia on HbA1c values among subjects without diabetes in University of Milano-Bicocca, Desio, Italy. Results showed that non-diabetic individuals with iron deficiency anemia presented significantly higher HbA1c measurements when compared to those obtained in non-anemic subjects [19]. Solomon., et al. studied the effect of iron deficiency anemia on HbA1c in diabetic patients at Tikor Anbessa specialized teaching hospital, Addis Ababa, Ethiopia among a total of 174 diabetic patients (87 with IDA and 87 without IDA) participated in the study and they found that HbA1c was significantly lower in IDA group $(6.18 \pm 1.57)$ compared to the control group. This difference might be due to different population (adults) and the different working definition by investigators who used different methods in detecting the level of HbA1c (by COBAS C 111 analyzer) as well as serum ferritin [20]. This study found a statistically significance correlation between duration of diabetes and $\mathrm{Hb}$ while there was no statistically significant correlation between duration of diabetes, serum iron and serum ferritin respectively. Thomas., et al. studied the prevalence of anemia as defined by decreased $\mathrm{Hb}$ concentration and reported $14 \%$ in those with 20 years duration of diabetes [21].

A study done by Ewa, reported that some significant inverse correlation in the MCH concentration in comparison with the chil- 
dren who have type 1 diabetes for more than 1 year. It has been shown that, when compared with those who had the disease for 1 to 3 years (FMD), those who have it for 4 to 6 years (SMD), and those who have it for more than 6 years (LD), more newly diagnosed type 1 diabetes (ND) children are characterized by the coexistence of at least 3 reduced values of the morphological blood parameters associated with iron-deficiency anemia $(26 \%, 17 \%, 7 \%$, and $3 \%$, respectively). At the same time, it has been found that the mean iron concentration in the blood serum of the ND group is significantly lower than that of the SMD and LD groups [22]. Sinha., et al. reported low value of $\mathrm{Hb} \mathrm{A} 1 \mathrm{c}$ level in patients with severe irondeficiency anemia so it may indicate that iron deficiency per se may elevate $\mathrm{HbA1c}$ level irrespective of the presence of anemia [23].

\section{Limitations of the Study}

Our study has some limitations that need to be acknowledge. The sample of patients were only conducting from a single center in a single time point. Small study sample reduces the power for external validity that means that results obtained cannot be generalized globally to all type 1 pediatric diabetic patients. The crosssectional design and observational nature of our study prevents any inferences of causality. Therefore, these data can only be used to generate some hypotheses that can be used in the future for a confirmatory study such as large randomized clinically controlled trials. Since this an observational study, many different techniques that can be applied to prevent or control for confounding factors could not be applied. The most commonly confounding factors encountered which were hard to measure and adjust for were dietary iron intake.

\section{Future Research Directions}

The relationship between anemia and HbA1c is sophisticated and can't be formulated due to variations among different clinical settings. Decisions of diagnosing and treating ype1 diabetes based lonely on low-threshold of HbA1c in children may lead to overdiagnosis and overtreatment. Another reliable novel biomarkers are needed that may be derived from advanced untargeted metabolomics and emerging advanced technologies. For example in a recent meta-analysis of genome-wide association studies, DUOX2 misense variant was detected in $14 \%$ of iron deficiency anemic patients and was associated with all iron metabolic biomarkers. was also suggested that that variant may increase the risk of IDA [24].

\section{Conclusion}

Iron-deficiency anemia is positively associated with increased HbA1c in type 1 diabetic children. So, the interpretation of HbA1c value should be interpreted with caution in newly diagnosed type 1 diabetic children with IDA and concomitant low diagnostic threshold of HbA1c. Furthermore, IDA should be treated first for proper use of $\mathrm{HbA1c}$ in monitoring glucose control in type 1 diabetic children.

\section{Conflict of Interest}

Authors declare no conflict of interest.

\section{Funding}

No funding received to conduct that study.

Ethical committee of Suez Canal University Hospital approved the study. Written informed consents were obtained from caregivers of children.

\section{Bibliography}

1. Punthakee Z., et al. "Definition, classification and diagnosis of diabetes, prediabetes and metabolic syndrome". Canadian Journal of Diabetes 42 (2018): S10-S15.

2. Mohan V and Pradeepa R. "The Global Burden of Diabetes and Its Vascular Complications". Mechanisms of Vascular Defects in Diabetes Mellitus: Springer (2017): 3-23.

3. Thomas MC., et al. "Screening for chronic kidney disease in patients with diabetes: are we missing the point?" Nature Clinical Practice Nephrology 4.1 (2018): 2-3.

4. Brugnara $\mathrm{C}$ and Eckardt KU. "Hematologic aspects of kidney disease”. In: Taal MW, ed. Brenner and Rector's The Kidney. 9th ed. Philadelphia: Saunders (2011): 2081-2120.

5. Thomas MC., et al. "Unrecognized anemia in patients with diabetes: a cross-sectional survey". Diabetes Care 26 (2003): 1164 Y1169.

6. Liang R and Ghaffari S. "Advances in understanding the mechanisms of erythropoiesis in homeostasis and disease". British Journal of Haematology (2016). 
7. Word Health Organization. Worldwide prevalence of anemia 1993-2005: WHO global database on anemia. Geneva: WHO (2008).

8. Halliwell B and Gutteridge JM. Free radicals in biology and medicine: Oxford University Press, USA (2015).

9. Thomas MC. "Anemia in diabetes: marker or mediator of microvascular disease?" Nature Clinical Practice Nephrology 3.1 (2007): 20-30.

10. Hassan A Shora., et al. "Assessment of Iron Deficiency Anemia as a Prognostic Factor for Childhood Asthma". EC Paediatrics 8.11 (2019): 127-136.

11. Enas Abbas., et al. "Nutritional Habits of Egyptian Primary School Female Children". CPQ Medicine 11.4 (2021): 01-17.

12. Salah N., et al. "Prevalence and type of anaemia in young Egyptian patients with type 1 diabetes mellitus". Eastern Mediterranean Health Journal 11 (2005): 959-967.

13. Akkermans MD., et al. "Iron status and its association with HbA1c levels in Dutch children with diabetes mellitus type 1". European Journal of Pediatrics 177.4 (2018): 603-610.

14. TARIM Ö., et al. "Effects of iron deficiency anemia on hemoglobin A1c in type 1 diabetes mellitus". Pediatric International 41.4 (1999): 357Y62.

15. Guo W., et al. "Increased levels of glycated hemoglobin a1c and iron deficiency anemia: A review". Medical Science Monitor 25 (2019): 8371-8378.

16. El-Agouza I., et al. "The effect of iron deficiency anaemia on the levels of haemoglobin subtypes: possible consequences for clinical diagnosis". Clinical and Laboratory Haematology 24.5 (2002): 285-289.

17. Madhu SV., et al. "Effect of iron deficiency anemia and iron supplementation on HbA1c levels - Implicationsfor diagnosis of prediabetes and diabetes mellitus in Asian Indians". Clinica Chimica Acta (2016).

18. Intra J., et al. "Glycosylated Hemoglobin in Subjects Affected by Iron-Deficiency Anemia". Diabetes and Metabolism Journal (2018): 42.
19. Solomon A., et al. "Effect of iron deficiency anemia on HbA1c in diabetic patients at Tikur Anbessa specialized teaching hospital, Addis Ababa Ethiopia”. BMC Hematology 19.1 (2019): 2.

20. Ewa M., et al. "The Occurrence of Iron-Deficiency Anemia in Children With Type 1 Diabetes". Journal of Investigative Medicine 62.6 (2014).

21. Sinha N., et al. "Effect of iron deficiency anemia on hemoglobin A1c levels". Annals of Laboratory Medicine 32 (2012): 17-22.

22. Dignass A., et al. "Limitations of Serum Ferritin in Diagnosing Iron Deficiency in Inflammatory Conditions". International Journal of Chronic Diseases 2018 (2018): 1-11.

23. Bell S., et al. "A genome-wide meta-analysis yields 46 new loci associating with biomarkers of iron homeostasis". Communications Biology 4 (2021): 156 (2021).

\section{Assets from publication with us}

- Prompt Acknowledgement after receiving the article

- Thorough Double blinded peer review

- Rapid Publication

- Issue of Publication Certificate

- High visibility of your Published work

Website: www.actascientific.com/

Submit Article: www.actascientific.com/submission.php

Email us: editor@actascientific.com

Contact us: +919182824667

Citation: Enas F Elngar, Hassan A Shora, Noran Bayoumi, Sylvias W Boulos and Enas S Abbas . "Iron-deficiency Anemia in Egyptian Type 1 Diabetic Children at Suez Canal University Hospital". Acta Scientific Medical Sciences 5.4 (2021): 67-73. 